\title{
Chapter 51
}

\section{ON THE SHEAR STRESS AT THE INTERFACE AND ITS EFFECTS IN TIE STRATIFIED FLOW}

\author{
Toshıo Iwasakı, Eng. D. \\ Professor of Civil Engineering \\ Tohoku University \\ Sendal, Japan
}

At the moderate velocity of the pure water which lies on the quiet salt water stable internal waves appear at the interfac in the stratified flow, and these waves will break and violated surface will arise if the velocity of the pure water may be increased. In this phase of phenomena the shear stress at the interface has the most important part. However observed ralues of this shear stress have not been reported in the systematıc style.

Experiments have been conducted in our laboratory since 1960. Some theoretical considerations could be served to get an empirical equation on the interfacial shear using experimental results and data presented by other researchers. This equation was as follows;

$$
\mathrm{k}_{\mathrm{f}}=3.940 \psi-0.8356
$$

In which $k_{f}$ is a coefficient of the internal shear stress $\sigma_{s}$ and is expressed as $T_{s} / \rho_{1} u^{2}$ where $\rho_{1}$ is the density of the upper layer, $u_{0}$ is the velocity and $\psi$ is $\left(\mathrm{Fr}^{i}\right)^{2} \mathrm{Re}^{\prime}$.

Free turbulent flow can be assumed to develope at the interface from the contact point of two layers. Owing to turbulence, Pure water was part1y mixed with salt water at rest. Weak flow was induced in the lower layer and the zone of Influence was developed downstream. Velocity and density distributions in both layers were derived theoretically following the methods of Tollmien and using the two dimensional convective diffusion equation, and were compared to the experimental values. Good coincidence was acquired.

The theory showed the zone of influence in the pure water diverged more pronouncedly and interfacial velocity decreased if the value of $k_{f}$ was large, when curves of the velocity

distribution had the remarkable gradient. However the diverging rate in the salt water was nearly constant for different values of $k_{f}$. For flow at the lower value of $\psi$ which was rather stable, theory showed the inverse flow in the salt water which was verified by experiments. In the case at the higher value of $\psi$, such reverse flow did not exist. And intermediate value of \% gave no solution for the salt layer which was caused presumably by the interfacial instabılity.

The density distribution in the lower transient zone was nearly linear in the ordinary density difference. In the extreme case of the remarkable density difference, the density came near asymptotically to that of the upper layer. 


\section{INTRODUCTION}

At an estuary, in case of weak mixing the pure water flow quietly over the stational salt water building up the stratified flow. The shape of the interface can be calculated if the shear stresses along the interface and along the riverbed can be reasonably estimated.

In 1949, Keulegan described phenomena around this interface very clearly. When the relative velocity between the two layers is small, the flow is laminar. However when this velocity is increased up to the formation of ridges and waves, the flow becomes turbulent. ${ }^{1)}$ This shows the shear stress at the interface is closely related to the interfacial waves in case of turbulent flow. In 1959, Bata derived formulae on frictional resistance at the interface employing the boundary layer theory. 2) It was the flow established on which he treatec but in the ordinary flumes in laboratories such established flow can be hardly realized and flow is usually non-established. This may be assumed the case also in the real flow.

In this paper another approach is tried to express the internal shear stress using some semi-theoretical considerations and the flow in the zone of establishment is analyzed making ust of the theory of free turbulence originated by Tollmien and of the corrvective-diffusion equation.

The theory presented herin may be considered casting some light upon phenomena in the stratified flow.

\section{THE COEFFICIENTS OF THE SHEARING STRESS AT THE INTERFACE}

Keulegan defined the number $\theta$ as the criterion of the interfacial instability in the stratified flow which was

$$
\theta=\left(\nu_{2} g \Delta p / \rho_{1}\right)^{1 / 3} / u_{0}
$$

in which $u$ was the relative velocity, $\rho_{1}$ was the density of the upper fluid, $\Delta \rho$ was the density difference, $\nu_{2}$ was the kinetic viscosity of the lower layer and $g$ was the accerelation of gravity.

Then following expressions hold;

$$
\begin{aligned}
& \psi=\frac{1}{\theta^{3}}=\left(F_{2}^{\prime}\right)^{2} \cdot R_{2}=\frac{16}{s} \cdot \frac{\lambda_{2}}{\lambda_{1}} \\
& \lambda_{1}=\left.\frac{8 \pi \nu_{2}}{s} \frac{\omega}{\left(u_{0}-\omega\right)^{2}}\right|_{\omega=\frac{u_{0}}{2}} \\
& \lambda_{2}=\frac{\pi u_{0}^{2}}{g^{\prime}}
\end{aligned}
$$


\& is introduced for the sake of convenzence. $F_{2}^{\prime}$ is the internal Froude number $u_{0} / \sqrt{g^{l} d_{2}}, R_{2}$ is the internal Reynolds number $u_{0} d_{2} / \nu_{2}$ in which $d_{2}$ is the depth of the lower layer. s is the sheltering coefficient and $\omega$ is the wave celerity on the interface. And $g^{p}$ is the reduced gravitational accerelation.

After Keulegan we can easily define $\lambda_{1}$ as the wave length of the stable internal waves in the viscous stratified flow and $\lambda_{2}$ as that in the irrotational stratified flow.

In turbulent flow, the critical value of $\theta$ is nearly equal to 0.018 . So the critical value of $\psi$ is 170 , and if we assume $s$ as 0.274 following Jeffereys, ${ }^{3)}$ the ratio $\lambda_{2} / \lambda_{1}$ is nearly equal to 3.00 which suggests the wave energy in the viscous flow is much smaller than that in the potential flow. When the value $\psi$ becomes larger, the internal waves are beggining to break and energy must be consumed in this breaking. Internal shear stress is that which cause energy 1oss. so, the coefficient $k_{f}$ which is $\tau_{s} / \rho_{1} u_{0}$ must also be correlated with the $\psi$ number.

In fig.1, data are plotted from experiments conducted in the Hydraulic Laboratory of Tohoku University since 1960, from experiments by $\mathrm{Dr}$.T.Hamada ${ }^{4}$ and from observations in River Ishikari. 5)

The relation between $k_{f}$ and $\psi$ is given as follows;

$$
k_{f}=3.940 \psi^{-0.8356}
$$

and certifies the assumption mentioned above.

\section{TURBULENT DIFFUSION IN THE STRATIFIED FLOW}

When the pure water with density $\rho_{1}$ flows over the still salt water with density $\rho_{2}$, there must take place momentum exchange by turbulent diffusion both in pure and salt water. In fig.2, zones of influence are assumed as lines $O B$ and $O C$. Then the horizontal velocity on the line $O B$ is equal to $u_{0}$, the incident velocity of the pure water and that on the line $O C$ is equal to zero. In the region between $O B$ and OC, velocity varies gradually, but the density is rather constant in the region between $O A$ and $O B$ and varies in the region between $O B$ and $O C$. This is because transfered water is easily diffused in the pure water flowing with the finite velocity $u_{0}$ but is not so easily diffused in the salt water which is stable.

In this connection we can assume as;

$$
\tau=\rho_{1} 1^{2}\left|\frac{d u}{d y}\right|^{2}
$$




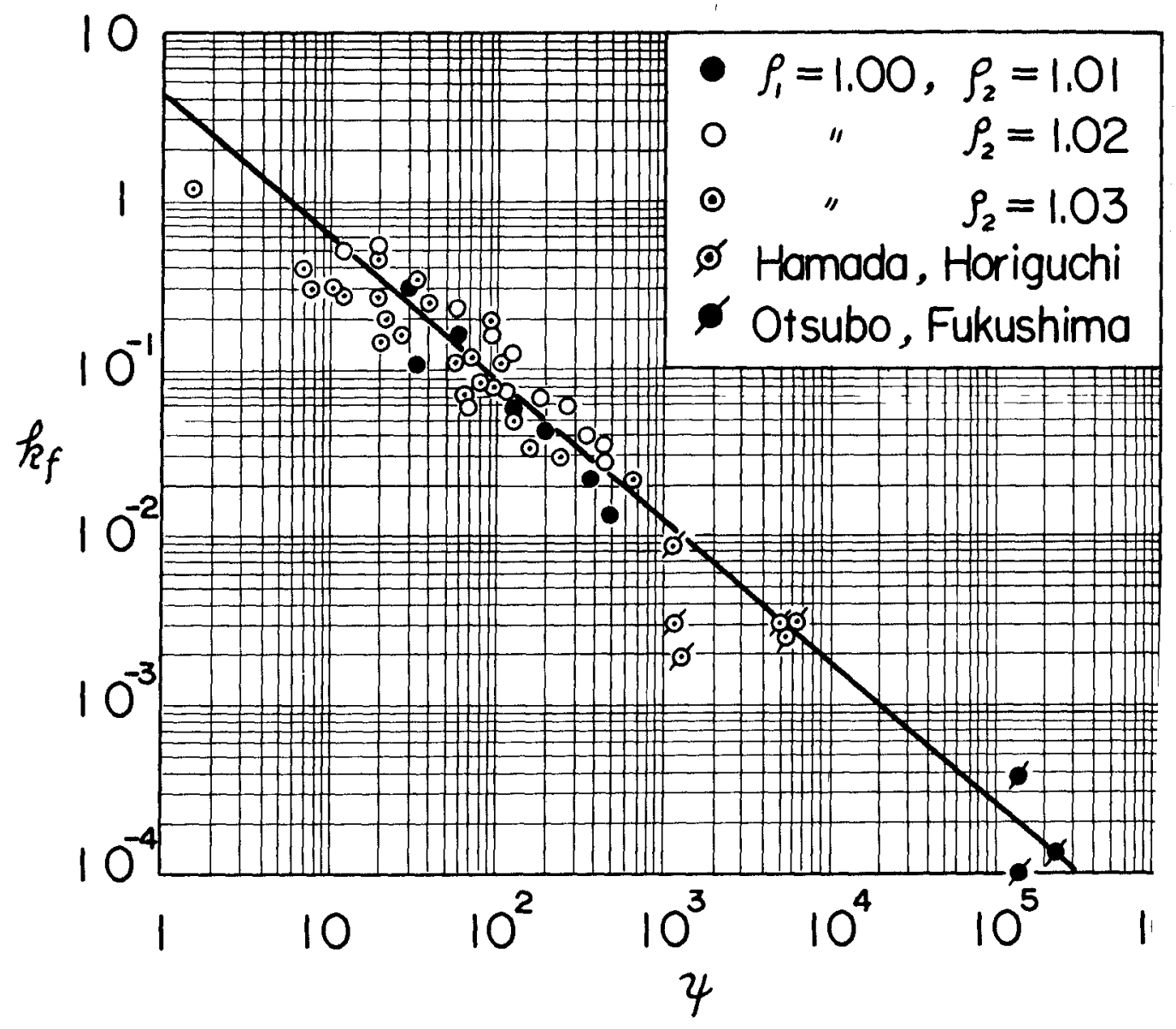

Fig.1. Relationship between $k_{f}$ and $\psi$. 


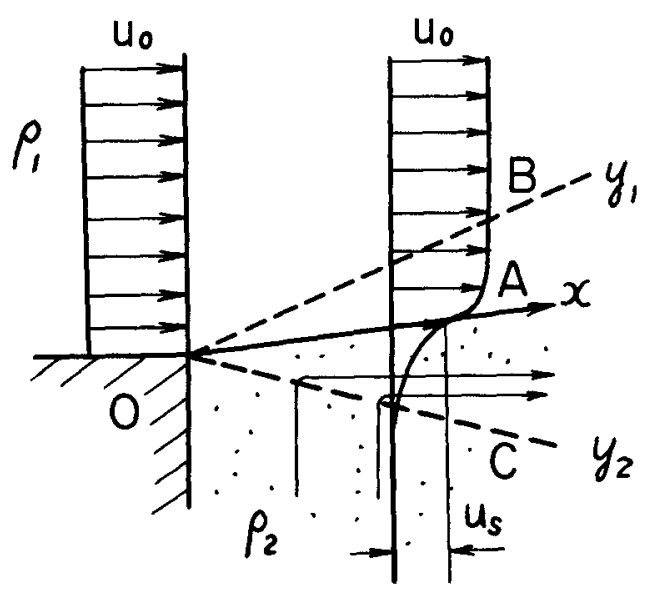

Fig.2. Illustration of the turbulent diffusion in the stratified flow.

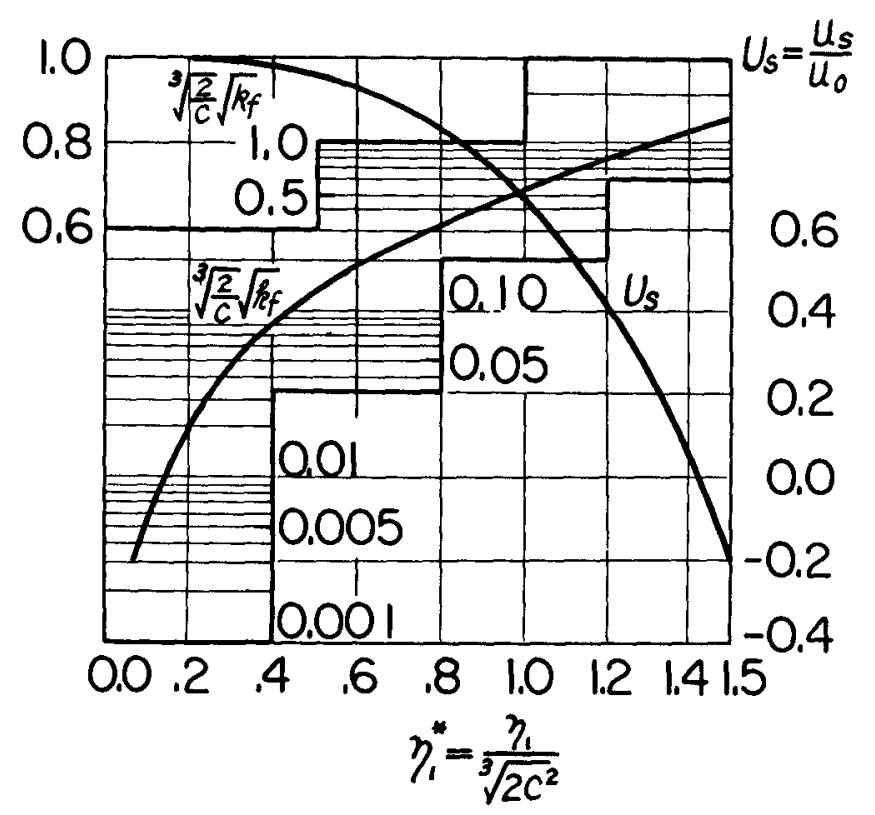

Fig. 3. Relationship between $U_{s}, \eta_{1}^{*}=\frac{\eta_{1}}{\sqrt[3]{2 c^{2}}}$ and $\sqrt[3]{\frac{2}{c}} \sqrt{k_{f}}$. 
where 1 is the Prandtl's mixing. length and is assumed proportiou to the horizontal distance from the point of contact 0 , that is $1=c x$ in which $c$ is constant.

FREE TURBULENT FLOW IN THE ZONE OF AOB

The equation of motion is

Now we put

$$
u \frac{\partial u}{\partial x}+v \frac{\partial u}{\partial y}=\frac{1}{\rho} \frac{\partial r}{\partial y}
$$

$$
U=\frac{u}{u_{0}}=f\left(\frac{y}{x}\right)=f(\eta)=F^{\prime}(\eta)
$$

as Tollmien in which $y$ is taken vertical to the interface OA. ${ }^{6)}$ The stream function $\psi$ is,

$$
\begin{aligned}
& \psi=\int f\left(\frac{y}{x}\right) d y=x \int f(\eta) d \eta=x F(\eta) \\
& \therefore V=\frac{v}{u_{0}}=-\frac{\partial \psi}{\partial x}=-F(\eta)+\eta F^{\prime}(\eta)
\end{aligned}
$$

Then eq. (7) can be rewritten as;

$$
F+2 c^{2} F^{\prime \prime \prime}=0
$$

in which we take $F " \neq 0$.

Boundary conditions are as follows;

$$
\begin{aligned}
& \text { at } \eta=\eta, \quad F(\eta)=\eta_{1}, F^{\prime}(\eta)=1, F^{\prime \prime}(\eta)=0 \\
& \text { at } \eta=0, \quad F^{\prime}(0)=\frac{u_{s}}{u_{0}}=U_{s}, F^{\prime \prime}(0)=\frac{\sqrt{k_{f}}}{c} \\
& u_{s} \text { is the velocity at of. }
\end{aligned}
$$

in which $u_{s}$ is the velocity at OA.

Now we put $\eta^{*}=\frac{\eta}{\sqrt[3]{2 c^{2}}}$, then eq. (11) can be reduced to

$$
F+\frac{d F}{d \eta *^{3}}=0
$$

and the boundary conditions are

$$
\begin{aligned}
& \text { at } \eta^{*}=\frac{\eta_{1}}{\sqrt[3]{2 c^{2}}}, \quad F\left(\eta_{1}^{*}\right)=\sqrt[3]{2 c^{2} \eta_{1}^{*}}, F^{\prime}\left(\xi_{1}^{*}\right)=\sqrt[2]{2 c^{2},} F^{\prime \prime}(\eta)=0 \\
& \text { at } \eta^{*}=0, \quad F^{\prime}(0)=\sqrt[3]{2 c^{2}} \cdot U_{s} \quad F^{\prime \prime}(0)=\sqrt[2]{4 c \sqrt{k f}} \\
& \text { From (13) and (14), we can get } \\
& F=A e^{-\eta^{*}}+B e^{\frac{\eta^{*}}{2} \cdot \cos \frac{\sqrt{3}}{2} \cdot \eta^{*}}+C e^{\frac{\eta^{*}}{2}} \cdot \sin \frac{\sqrt{3}}{2} \eta^{*}
\end{aligned}
$$

and

$$
\begin{aligned}
U=\frac{u}{u_{0}}=-A e^{-\eta^{*}} & +\frac{1}{2}(B+\sqrt{3} C) e^{\frac{\eta^{*}}{2}} \cdot \cos \frac{\sqrt{3}}{2} \eta^{*} \\
& -\frac{1}{2}(\sqrt{3} B-C) e^{\frac{\eta^{*}}{2}} \cdot \sin \frac{\sqrt{3}}{2} 3^{*}
\end{aligned}
$$


where,

$$
\begin{aligned}
& A=\alpha e^{\eta_{1}^{*}}\left(\eta_{1}^{*}-1\right) \\
& \beta=\alpha e^{-\eta_{1}^{*}}\left[\left(1+2 \eta_{1}^{*}\right) E_{c}-\sqrt{3} E_{s}\right] \\
& c=\alpha e^{-\eta_{1}^{*}}\left[\sqrt{3} E_{c}+\left(1+2 \eta_{1}^{*}\right) E_{s}\right] \\
& E_{c}=e^{\frac{\eta_{1}^{*}}{2}} \cos \frac{\sqrt{3}}{2} \eta_{1}^{*}, E_{s}=e^{\frac{\eta^{*}}{2}} \sin \frac{\sqrt{3}}{2} \eta_{1}^{*} \eta_{1}^{*}-\eta / \sqrt[3]{2 c^{2}} \\
& \alpha=1 / 3 \cdot \sqrt[3]{2 c^{2}} .
\end{aligned}
$$

FREE TURBULENT FLOW IN THE ZONE OF AOC

In the zone of $A O C$, the density varied gradually. Then we must take $\rho$ in eq. ( 7 ) as a variable.

If we assume eq.(8) also hold in this zone, we can get from eq. (6) as,

$$
\frac{1}{u_{0}^{2}} \frac{\partial \tau}{\partial y}=\frac{c^{2} F^{\prime \prime}}{x}\left(F^{\prime \prime} \rho^{\prime}+2 F^{\prime \prime \prime} \rho\right)
$$

in which, $p^{\prime}=d \rho / d \eta$.

Then from eq.(7), we can derive

$$
F+2 c^{2} F^{\prime \prime \prime}+c^{2} \frac{\rho^{\prime}}{\rho} F^{\prime \prime}=0
$$

and the boundary conditions are

$$
\begin{array}{lll}
\text { at } \eta=0, & F^{\prime}(0)=U_{S}, & F^{\prime \prime}(0)=\sqrt{k+} / c \\
\text { at } \eta=\eta_{2}, & F^{\prime}\left(\eta_{2}\right)=0, & F^{\prime \prime}\left(\eta_{2}\right)=0
\end{array}
$$

in which $\eta_{2}$ denotes the lower boundary $O C$.

The two-dimensional convectuve diffusion equation is,

$$
u \frac{\partial S}{\partial x}+v \frac{\partial S}{\partial y}=\frac{\partial}{\partial x}\left(E_{x} \frac{\partial S}{\partial x}\right)+\frac{\partial}{\partial y}\left(E_{y} \frac{\partial S}{\partial y}\right)
$$

where $s$ is the salinity and $E_{x}$ and $E_{y}$ are the coefficients of horizontal and vertical eddy diffusivity.respectively. In turbulent flow, molecular diffusivity is negligible and eddy diffusivity is predominant. So, we can assume $E_{x}$ and $E_{y}$ as $c^{2} x^{2}(\partial u / \partial y)$ as eddy viscosity.

The density $\rho$ and the salinity $s$ are related by;

$$
\rho=\rho_{1}(1+a s)
$$

And if we take $\rho$ as a function of $\eta$ like $u$, the following equation can be reduced from eq. (21),

$$
\frac{\rho^{\prime \prime}}{\rho^{\prime}}=-\frac{1}{F^{\prime \prime}}\left[F^{\prime \prime}+\frac{1}{c^{2}} \cdot \frac{\eta}{1+\eta^{2}} \cdot F^{\prime}\right]
$$

From eq. (19)

$$
p=-\frac{\rho}{c^{2} F^{\prime \prime}}\left(F+2 c^{2} F^{\prime \prime \prime}\right)
$$

in which $\mathrm{p}=\frac{d \rho}{d \eta}=\rho^{\prime}$. And if we differentiate it, we get 


$$
\frac{d p}{d p}=-\frac{1}{c^{2} F^{\prime \prime}}\left(F+2 c^{2} F^{\prime \prime \prime}\right)
$$

As $\rho^{\prime \prime} / \rho^{\prime}=\mathrm{dp} / \mathrm{d} \rho$, from (23) and (24)

$$
\begin{aligned}
& \frac{1}{c^{2}}\left(F+2 c^{2} F^{\prime \prime}\right)=F^{\prime \prime \prime}+\frac{1}{c^{2}} \frac{\eta}{1+\eta^{2}} F^{\prime} \\
& c^{2} F^{\prime \prime \prime}+F^{\prime} \frac{\eta}{1+\eta^{2}}+F=0
\end{aligned}
$$

If we put $\overline{\eta^{*}}=\frac{\eta}{\sqrt[3]{c^{2}}}(27)$, then eq. (26) can be reduced to,

$$
\frac{d^{3} F}{d \bar{\eta}^{*^{3}}}+\frac{\overline{\eta^{*}}}{1+c^{* / 3} \bar{\eta}^{2}} \frac{d F}{d \bar{\eta}^{*}}+F=0
$$

The boundary conditions (20) are reduced to,

$$
\begin{aligned}
& \text { at } \bar{\eta}^{*}=0, \frac{d F}{d \eta^{*}}=\sqrt[3]{c^{2}}, U_{s}, \quad \frac{d^{2} F}{d \bar{\eta}^{2}}-\sqrt[3]{c} \sqrt{k t} \\
& \text { at } \overline{\eta^{*}}=\overline{\eta_{2}^{*}}=\frac{\eta_{2}}{\sqrt[3]{c^{2}}}, \quad \frac{d F}{d \eta^{*}}=0, \quad \frac{d^{2} F}{d \bar{\eta}_{*}^{2}}=0
\end{aligned}
$$

If we assume $F$ as the power series of $\bar{\eta}^{*}$, and if we define 1 ts coefficients by (28) and (29), we can get its solution as;

$$
F=A_{0}+A_{1} \bar{\eta}^{*}+A_{2} \bar{\eta}^{*^{2}}-\frac{A_{0}}{6} \bar{\eta}^{*^{3}}-\frac{A_{1}}{12} \bar{\eta}^{*}-\frac{A_{2}}{20} \bar{\eta}^{-5}
$$

in which

and

$$
\begin{aligned}
& A_{0}=\frac{k\left(24+4 y+r^{2}\right)}{4 \bar{\eta}_{2}^{*}(6+y)}, A_{1}=U_{1}^{*} A_{2}=\frac{k}{2}, \quad U^{*} \sqrt[3]{c^{2}} U_{s} \\
& K=\sqrt[3]{c} \sqrt{k f}, \quad Y=\left(\bar{\eta}_{2}^{*}\right)^{3}
\end{aligned}
$$

$$
\begin{aligned}
\frac{u}{u_{0}} & =U=\frac{d F}{d \eta}=\frac{1}{\sqrt[3]{c^{2}}} \cdot \frac{d F}{d \eta^{*}} \\
& =U_{s}\left(1+W \eta^{*}-\frac{1}{\sqrt[3]{4}} W f(y)\left(\eta^{*}\right)^{2}-\frac{2}{3}\left(\eta^{*}\right)^{3}-\frac{W}{4}\left(\eta^{*}\right)^{4}\right) \\
W & =\frac{1}{U_{s}} \sqrt[3]{\frac{2}{c}} \sqrt{k_{f}}, \quad f(y)=\frac{24+4 y+y^{2}}{4 \bar{\eta}_{2}^{*}(6+y)}=\frac{24+4 y+y^{2}}{4 \sqrt[3]{2}(6+y) \eta_{2}^{*}} \\
y & \approx\left(\bar{\eta}_{2}^{*}\right)^{3}=2\left(\eta_{2}^{*}\right)^{3}
\end{aligned}
$$

in which we use the relation of $\bar{\eta}^{*}=\sqrt[3]{2} 3^{*}=\frac{3}{\sqrt[3]{c^{2}}}$ for $1 t$ may be convenience that equations (16) and (32) have either the same independent variable. 
THE EFFECTS OF $\mathrm{k}_{f}$ UPON THE INTERFACIAL VELOCITY

AND BOUNDARTES OF MIXING ZONES

From eq.(14), the non-dimensional interfacial velocity can be written as;

$$
U_{s}=\frac{1}{\sqrt[3]{2 c^{2}}} F^{\prime}(0)
$$

Using eq. (15) and (17), eq. (34) is reduced to;

$$
U_{s}=\frac{U_{s}}{U_{0}}=\frac{1}{6 \alpha}(-2 A+B+\sqrt{3} C)
$$

Also from eq. (14),

$$
\sqrt[3]{4 C} \sqrt{k f}=F^{\prime \prime}(0)
$$

And this equation can be rewritten as

$$
\sqrt[3]{4 C} \sqrt{k f}=A-\frac{B}{2}+\frac{\sqrt{3}}{2} C
$$

in these equations, A,B,C and $\alpha$ are expressed in eq.(17).

In fig. 3, the relationship between $U_{s}$ and $\eta_{1}^{*}$ is given in the normal scale. And that between $\sqrt[3]{\frac{2}{c}} \sqrt{k_{f}}$ and $\eta_{1}^{*}$ is glven in the semi-logarithmic scale. It is shown that if $k_{f}$ becomes

large, the upper zone of mixing diverges more rapldly because $\eta_{1}$ is the ratio of the value $y$ at the upper boundary oB and the distance $x$ from the point of contact.

The interfacial velocity $U_{S}$ decreases when the coefficient of the shear stress increases.

Following Tollmien, we may assume the value $c$ as 0.0174 . The critucal value of $\psi$ is 170 as cited above. Then, from eq. (5) $\mathrm{k}_{\mathrm{f}}$ is 0.055 . And from fig.3 the critical value of $\eta_{1}^{*}$ can be given as 1.40. In this case the value $U_{s}$ is zero, and in case of the value $3 \sqrt{\frac{2}{c}} \sqrt{k_{f}}$ lower than $1.14, U_{s}$ is negative as in fig。3, which is meaningless from the physical aspect and also substantiates the critical condition stated by Keulegan. from eq. (29);

Using eq. (30) the following relation can be derived

$$
\frac{U_{s}}{\sqrt[3]{\frac{2}{c}} \sqrt{k_{f}}}=-\frac{3}{4} \cdot \frac{4+y}{6+y} \cdot \eta_{2}^{*} ; y=2\left(\eta_{2}^{*}\right)^{3}
$$

where $\eta_{2}^{*}=\eta_{2} / \sqrt[3]{20^{2}}$ and $\eta_{2}$ is the ratio of the value $y$ at the lower boundary $O C$ and $x$. As expressed in fig.4, we know 


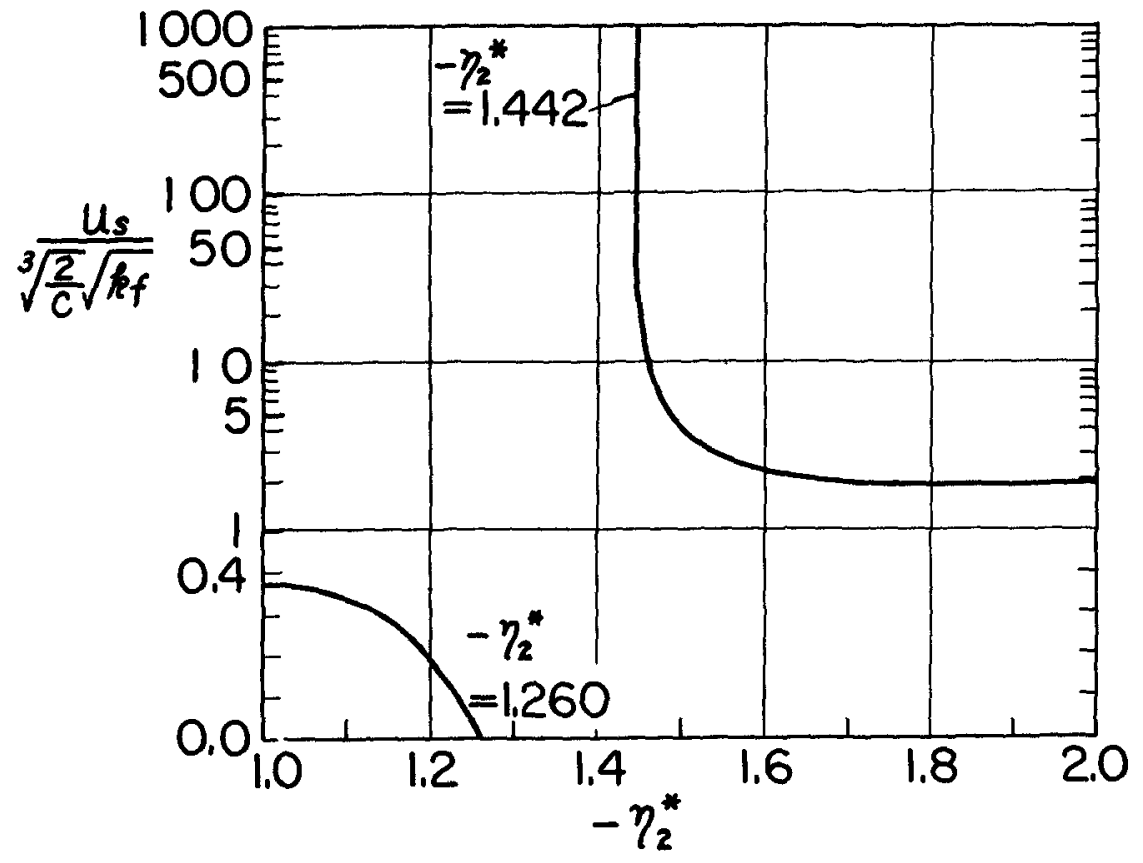

Fig. 4. Relationship between $\frac{U_{s}}{\sqrt[3]{\frac{2}{c} \sqrt{k_{f}}}}$ and $-\eta_{2}^{*}$.

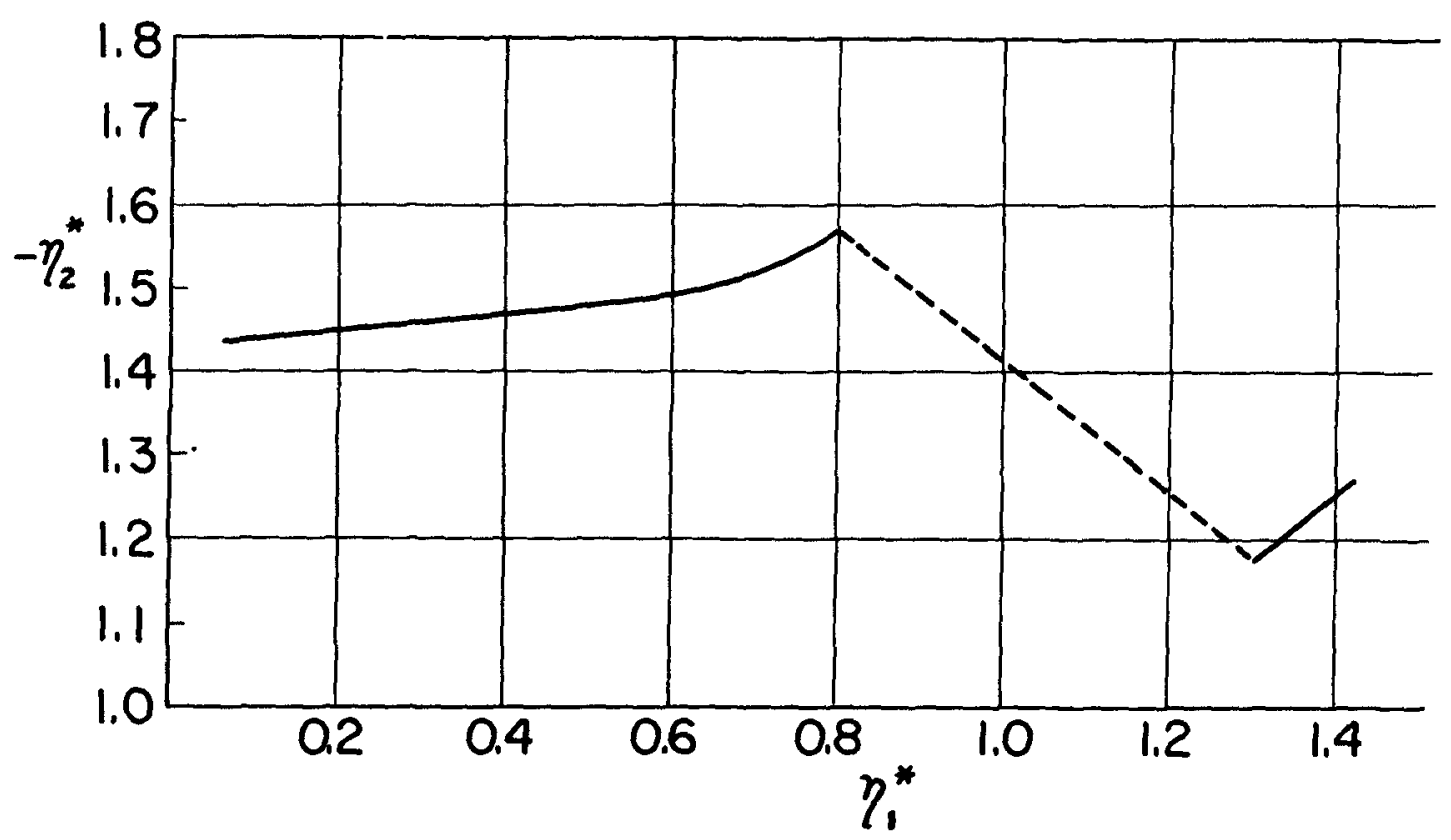

Fig. 5. Relationship between $\eta_{1}^{*}$ and $-\eta_{2}^{*}$. 
that no real solution of the lower boundary can be given for the values of $u_{s} / \sqrt[3]{\frac{2}{c} \sqrt{k_{f}}}$ between 0.368 and 1.792 .

From fig. 3 , values of $U_{s}$ and $\sqrt[3]{\frac{2}{c} \sqrt{k_{f}}}$ can be taken for any value of $\eta_{1}^{*}$ and their ratio gives the related value of $\eta_{2}^{*}$ in fig.4. Fig. 5 shows the relationship between $\eta_{1}^{*}$ and $\eta_{2}^{*}$ thus acquired. In which, the dotted line shows the region where no solution can be given for the lower boundary as cited above. And also it is shown that there'is no solution for the value of $\eta_{1}^{*}$ higher than 1.40 .

\section{VELOCITY DISTRIBUTIONS IN THE ZONES OF INFLUENCE}

In fig. 6 , the velocity distributions are presented from eq. (16) and (32). The ordinates is taken for $\eta^{*} / \eta_{1}^{*}$, so the velodity at the interface is shown at the horizontal axis. Four curves are shown. The curve for $k_{f}=0.055$ which is critical as cited above shows the remarkable gradient of the velocity distribution in the pure water and a reverse flow in the salt water which is the phenomenon that has been observed frequently in the small scale flume. The curve for $k_{f}=0.01$ presents no solution in the velocity field of the lower 1 ayer. The curve for 0.001 of the value of $k_{f}$ is an example for the large scale flume and the fourth curve is for $k_{f}=0.0001$ which is observed in the real estuaries. 5) In these cases there happens no reverse flow and the zone of influence in the lower layer spreads remarkably in contrast to that in the upper 1 ayer which has also been observed in an estuary.

\section{DENSITY DISTRIBUTIONS IN THE ZONES OF INFLUENCE}

$$
\text { From eqs. (24) and (25), we get; }
$$

then we can deduce,

$$
\frac{p}{\rho}=\frac{d p}{d p}
$$

$$
\rho=k e^{c \eta}
$$

and the boundary conditions are

$$
\text { at } \eta=0, \rho=\rho_{0} ; \text { at } \eta=\eta_{2}, \quad \rho=\rho_{0}(1+\varepsilon)
$$

then from (40) and (41), we can derive as;

$$
1+\alpha \varepsilon=(1+\varepsilon)^{n / \eta_{2}}
$$




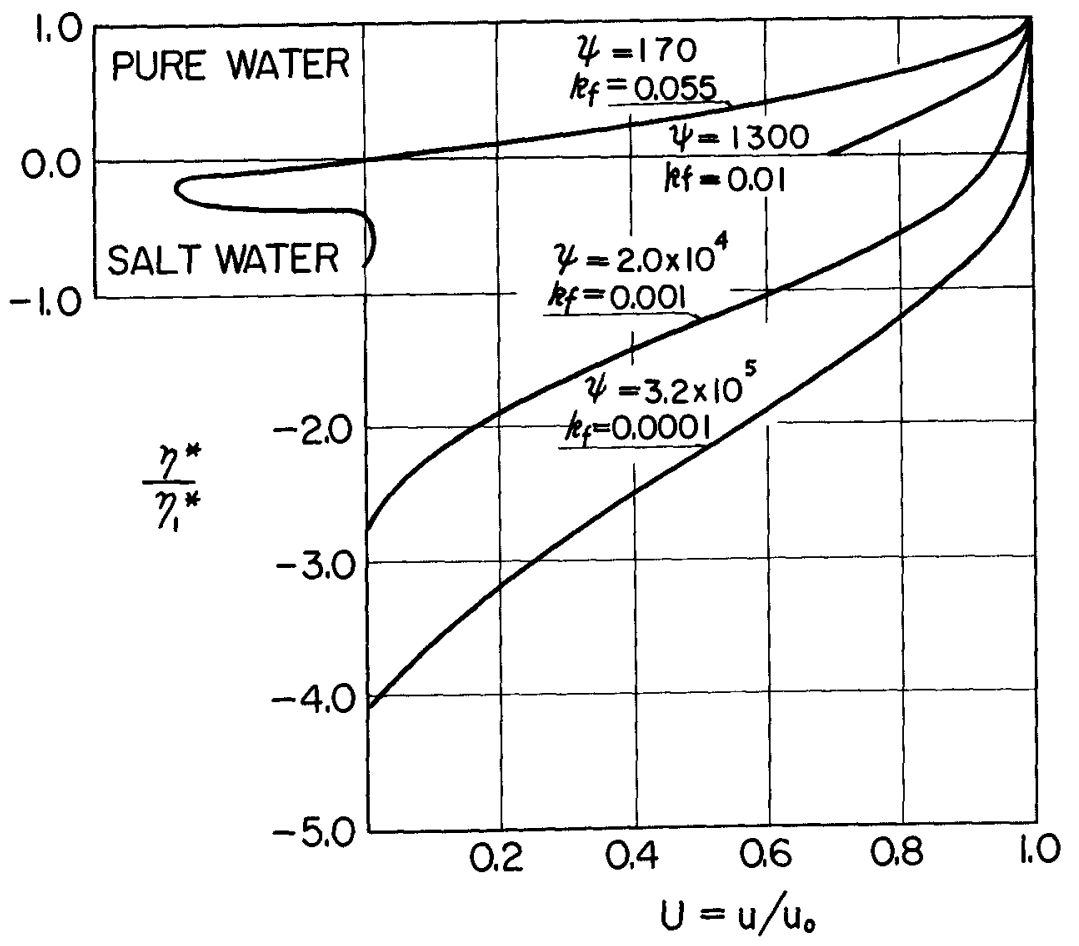

Fig.6. Velocity distributions in the zones of influence.

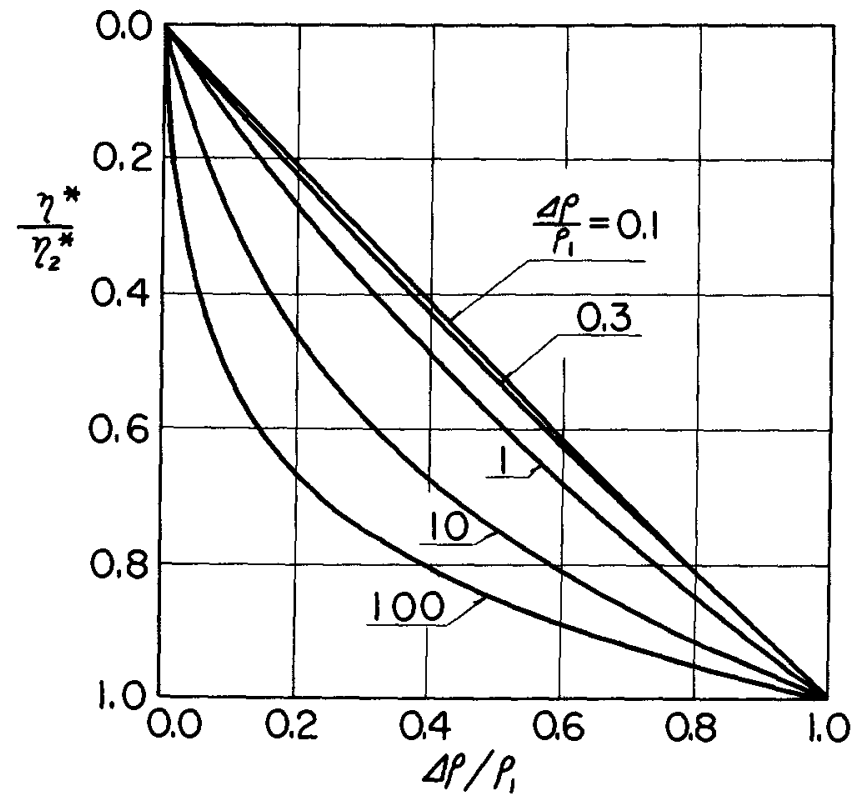

Fig.7. Density distributions in the lower zones of influence. 
In which $\alpha=\rho-\rho_{1} / \rho_{2}-\rho_{1}$.

Fig.7 shows the density distributions in the lower zones. For the values of $\Delta \rho / \rho_{1}$ between 0.1 and 0.3 , which are popular cases for the estuary, curved are nearly 11near. However If $\Delta \rho / \rho_{1}$ should be as large as 10 or 100 , density distributio might deviate remarkably from the linear one.

\section{CONCLUSIONS}

In comparison with experıments conducted in the Ifydraulic Laboratory in Tohoku University, the relation between $\sqrt[3]{\frac{2}{c} \sqrt{k_{f}}}$ and $U_{s}$ is reasonably hold and lower zone of influence was not distinctly recognized in the experiments in cases of $\mathrm{U}_{\mathrm{s}} / \sqrt[3]{\frac{2}{\mathrm{c}} \sqrt{\mathrm{k}_{\mathrm{f}}}}$ between 0.513 and 1.420 , which substantiated the above theory, If we assume c as 0.174 .

However values of $\eta^{*}$ derived from this theory were seemec to be rather high compared to the actual one. And in the experiments the density distributions had minute wavy configurations over the linearized appearance. The eddy diffusivity must be observed in detail for horizontal or vertical direction.

However the theory presented herin seemed to cast much light upon phenomena in the stratified flow.

\section{ACKNOWLEDGEMENT}

The auther wishes to acknowledge his appreciation to Professor T. Kataoka of Twate Unıversity for his precise execution of experiments as a visiting professor and to staffs of iydraulic Laboratory of Tohoku University for their efforts in the experiments.

\section{REFERENCES}

1. Keulegan,G.F. (1949). Interfacial instab1lity and mixing In stratified flow: Jour. Research Nat. Bur. Stand. vol. $43, \mathrm{p} 487, \mathrm{RP} 2040$

2. Bata,G.L.(1959). Frictional resistance at the interface of density currents: Proc. 8th Congress-Montreal, I.A.H.R. 12-C

3. Jeffereys, H. (1925)(1926). On the formation of water waves by wind: Proc. Roy. Soc. London A, vol.107 and 110 
4. Hamada, T. (1960). On the behaviour of the salt wedge: Proc. 7 th Conf. Jap. Coast. Eng.,p.163(in Japanese)

5. Otsbo,K. and Fukushima,H.(1959). Density currents in a river mouth with a small tidal range: Proc. 8th CongressMontreal, I.A.H, R. 4-C

6. Tollmien,w.(1926). Berechnung turbulenter Ausbreitungsvorgänge: Z.a.M.M., Bd. 6, p.468 